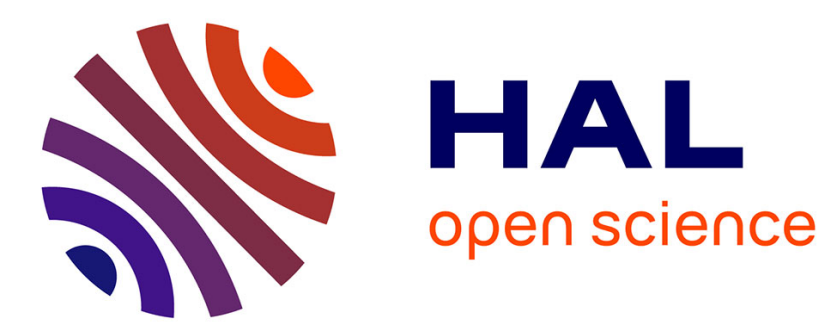

\title{
Moment-duration scaling of Low-Frequency Earthquakes in Guerrero, Mexico
}

\author{
Gaspard Farge, Nikolai M Shapiro, William B Frank
}

\section{To cite this version:}

Gaspard Farge, Nikolai M Shapiro, William B Frank. Moment-duration scaling of LowFrequency Earthquakes in Guerrero, Mexico. Journal of Geophysical Research: Solid Earth, 2020, 10.1029/2019JB019099 . hal-02879205v2

\section{HAL Id: hal-02879205 \\ https://hal.science/hal-02879205v2}

Submitted on 10 Jul 2020

HAL is a multi-disciplinary open access archive for the deposit and dissemination of scientific research documents, whether they are published or not. The documents may come from teaching and research institutions in France or abroad, or from public or private research centers.
L'archive ouverte pluridisciplinaire $\mathbf{H A L}$, est destinée au dépôt et à la diffusion de documents scientifiques de niveau recherche, publiés ou non, émanant des établissements d'enseignement et de recherche français ou étrangers, des laboratoires publics ou privés. 


\title{
Moment-duration scaling of Low-Frequency Earthquakes in Guerrero, Mexico
}

\author{
${\text { Gaspard Farge }{ }^{1 *} \quad \text { Nikolaï M. Shapiro }}^{2,3} \quad$ William B. Frank ${ }^{4,5}$ \\ 1 Université de Paris, Institut de Physique du Globe de Paris, CNRS,F-75005 Paris, France \\ 2 Schmidt Institute of Physics of the Earth, Russian Academy of Sciences, Moscow, Russia \\ 3 Institut de Sciences de la Terre, Université Grenoble Alpes, CNRS (UMR5275), Grenoble, France \\ ${ }^{4}$ Department of Earth Sciences, University of Southern California, Los Angeles, CA, USA \\ ${ }^{4}$ Department of Earth, Atmospheric and Planetary Sciences, Massachusetts Institute of Technology, Cambridge, \\ Massachusetts, USA
}

Manuscript first published in Journal of Geophysical Research: Solid Earth, the $24^{\text {th }}$ June 2020.

\begin{abstract}
Low-frequency earthquakes (LFEs) are detected within tremor, as small, repetitive, impulsive low-frequency $(1-8 \mathrm{~Hz})$ signals. While the mechanism causing this depletion of the high-frequency content of their signal is still debated, this feature may indicate that the source processes at the origin of LFEs are different from those for regular earthquakes. Key constraints on the LFE-generating physical mechanisms can be obtained by establishing scaling laws between their seismic moment and source durations. Here we apply a simple spectral analysis method to the S-waveforms of LFEs from Guerrero, Mexico to measure their seismic moments and corner frequencies, a proxy to source duration. We find characteristic values of $M_{0} \sim 3 \times 10^{12}$ N.m $\left(M_{w} \sim 2.3\right)$ and $f_{c} \sim 3.0 \mathrm{~Hz}$ with the corner frequency very weakly dependent on the seismic moment. This moment-duration scaling observed for Mexican LFE is similar to one previously reported in Cascadia and is very different from the established one for regular earthquakes. This suggests that they could be generated by sources of nearly constant size with strongly varying intensities. LFEs do not exhibit the self-similarity characteristic of regular earthquakes, suggesting that the physical mechanisms at their origin could be intrinsically different.
\end{abstract}

\section{Plain language summary}

Low-frequency earthquakes are unusual, small earthquakes that are detected on the deep end of plate boundary faults. They occur during periods of slow slip, when the plates start sliding against each other much slower than during earthquakes, but long enough to release the energy that might otherwise have caused large earthquakes. Thus, they carry precious information about the fault behavior while slow slip is active.

In this study, we analyze a selection of low-frequency earthquakes from a slow slip zone of the Mexican subduction, beneath the state of Guerrero. We find that their magnitude is on average higher than in other regions where they have been detected $\left(M_{w} 1.5--3\right)$. We also find that whatever the magnitude of these events, they always last about $0.3 \mathrm{~s}$.

On the contrary, regular earthquakes are known to last longer when they grow larger, and lowfrequency earthquakes in Nankai (Japan) appear to behave similarly. We discuss a physical mechanism explaining such a discrepancy with regular earthquakes and such strong regional variations. The implication of crustal fluids circulating at extremely high pressure in between plates might be one of the key components of this mechanism, as it often is for slow slip processes.

${ }^{*}$ farge@ipgp.fr 


\section{Contents}

\begin{tabular}{lll}
\hline & Introduction & 3
\end{tabular}

\begin{tabular}{|ll|}
2 & Low-frequency earthquakes in Guerrero, Mexico \\
\hline
\end{tabular}

\begin{tabular}{|ll|}
\hline 3 & Selection of LFEs with high-quality waveforms
\end{tabular}

\begin{tabular}{|lll|}
\hline & Characterizing seismic moment and corner frequency & 6
\end{tabular}

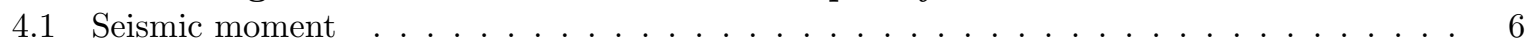

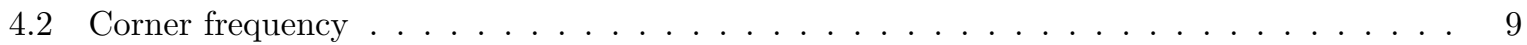

5 Results 9

$5.1 \quad$ Low-frequency earthquakes source parameters: seismic moment, corner frequency . . . . . 9

5.2 Moment-duration scaling $\ldots \ldots \ldots \ldots \ldots \ldots \ldots \ldots$

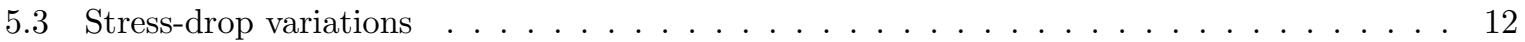

$\begin{array}{lll}6 & \text { Discussion and interpretation of the observed scaling law } & 12\end{array}$

6.1 Scaling laws of earthquakes, slow earthquakes and LFEs . . . . . . . . . . . . . . . 12

6.2 Potential physical mechanisms accounting for magnitude-independent duration of LFEs . 13

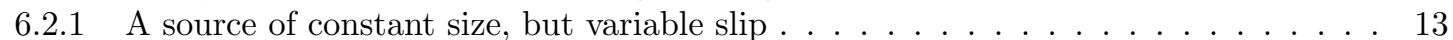

$6.2 .2 \quad$ Potential effects of fluid circulation and high fluid pressures $\ldots \ldots \ldots \ldots$. . . . . . 13

$\begin{array}{lll}7 \text { Conclusions } & 14\end{array}$

\begin{tabular}{|l|l|l|l|l|l|l|}
\hline A Effects of bandpass filtering on events corner frequencies & 15
\end{tabular}

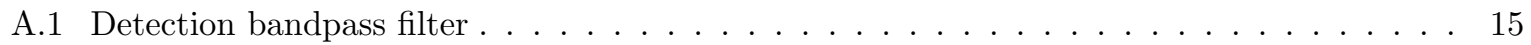

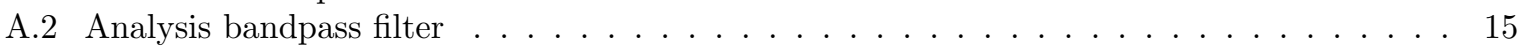

\begin{tabular}{|ll}
\hline B Moment-frequency distribution of LFEs & 16
\end{tabular} 


\section{Introduction}

Low-frequency earthquakes (LFEs) are observed in association with volcanic and tectonic processes as impulsive, coherent wave arrivals with discernible $\mathrm{S}$ and sometimes $\mathrm{P}$ phases and a low-frequency content (1-8 Hz) relative to their small magnitudes. Tectonic LFEs have been documented in various subduction zones $[e . g[7,19,63]$ and on strike-slip plate boundaries [e.g[12, 62]. They are detected in the transitional zones just beneath the regular seismogenic portions of the faults and are often associated with zones of relatively low shear-wave velocity, high $V_{P} / V_{S}$ ratio, low quality factor, all indicators of fluid rich media [4, 7, 63. LFE activity is closely correlated in time and space with slow slip activity, and is thus used to track the spatial extent of slow slip phenomena [26, 30, 53, 64, but also to detect short-duration and low-amplitude slow slip events that fall below the detection threshold for techniques relying on geodetic data [17, 25, 46].

In analogy with volcanic tremor, it was first suggested that tectonic tremors are generated by fluids circulating in the vicinity of the fault zone [39, 40, 49. Subsequent research based on the characteristics of tectonic tremors and LFEs signals - such as dominant S-wave content and shear double-couple mechanisms [7, 19, 33, 65, and association with slow slip events [24, 43, 57, — built empirical support for the hypothesis that they are generated by shear slip on the fault.

LFEs are a member of the "slow earthquake" class of phenomena, regrouping events which seem to be associated with slow slip processes on faults [6. Those events exhibit a wide range of durations (from slightly less than $1 \mathrm{~s}$ for LFEs, up to nearly a year for slow slip events) and magnitudes (from around $M_{w} 1$ up to $\left.M_{w} 7.5\right)$. When accounted as a one family of fault slip processes, their seismic moment $M_{0}$ appears to be directly proportional to their duration $T$ [32, whereas regular earthquakes align along a $M_{0} \propto T^{3}$ scaling law [2, 29, 54] due to the self-similarity of the rupture process [38. However, considering slowearthquakes as one entity relies on bridging two different scales of observations: geodetic observations of large-scale, long-term (sub-daily at minima) slow slip events and seismic observations of short term events as LFEs, and longer events (30-60 s) called very-low-frequency earthquakes (VLFEs). There seems to be evidence that the shortest slow slip transients measured to this day align along a self-similar momentduration scaling $M_{0} \propto T^{3}$ [23, and that only at the largest scales it would transition to a $M_{0} \propto T$ scaling law 28. This observation isolates LFEs and VLFEs from the bulk of geodetic observations of slow slip. It brings into question whether they are simply smaller scale manifestations of slow slip, or they have a more complex coupling relationship with it. In particular, LFEs co-occur with slow slip events, and investigating their properties provides key insight to understanding fault state, geometry of activation and overall rupture process during slow slip activity [5, 13, 21, 30, e.g.].

In this study, we investigate the moment-duration relationship for LFEs in Guerrero, Mexico. As for regular earthquake, evaluating how moment release scales with source duration is key to understand the dynamics of the process generating LFEs. Similar studies have already been carried out in regions witnessing tremor and slow slip. In Cascadia, LFEs source duration has been found to be very weakly dependent on their seismic moment, scaling along $M_{0} \propto T^{10}[8]$. On the other hand, in Nankai, LFEs exhibit a self-similar behavior, scaling along $M_{0} \propto T^{3}$ [66. To our knowledge this is the first published work to measure source parameters for LFEs in Guerrero, Mexico. We start with a large LFE catalog compiled by [20] and select those whose waveforms have a sufficiently high quality to measure their seismic moments and corner frequencies. The latter are estimated with two different methods to check the robustness of the obtained results. Similar to the study of 8 for LFEs in Cascadia, we find that for the LFEs in Guerrero the corner frequency is very weakly dependent on the seismic moment.

\section{Low-frequency earthquakes in Guerrero, Mexico}

Slow-slip events (SSE), tectonic tremor, and low-frequency earthquakes have been observed in the state of Guerrero, Mexico, in the subduction zone of the Cocos plate under the North American plate [19, 43, 51]. They have been detected in the Guerrero seismic gap - a corridor extending from Acapulco to Mexico City - that has not witnessed major earthquakes $\left(M_{w}>7\right)$ for more than 100 years [42. This longer recurrence time of major earthquakes compared to adjacent regions can be related to the large SSEs that accommodate a significant fraction of the plates convergence in the Guerrero seismic gap 56 .

We use the data collected during the Meso-American Seismic Experiment (MASE) [31, 52, between 1 January 2005 and 15 April 2007. Following the work of [18], we selected a subset of 10 stations out of the 100 total based on their high signal-to-noise ratio (SNR), the continuity in time of their records, and their proximity to the LFE activity (see Figure 1). 20] used this dataset and the method described in [18 to compile a large catalog of 1.8 million LFEs grouped in 1120 families of repeating events. We 

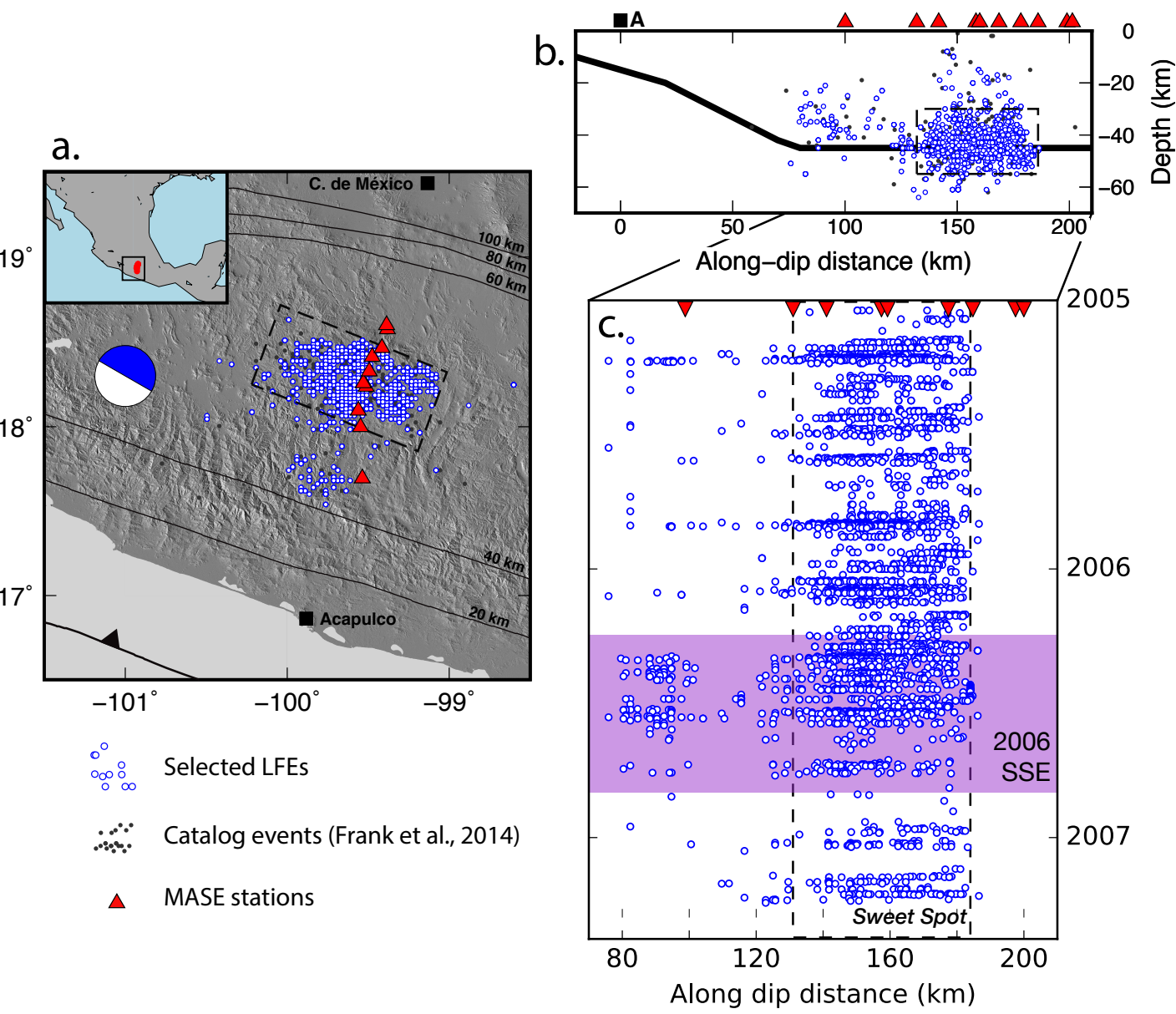

Figure 1: Spatial and temporal distribution of the selected LFEs - Selected LFEs are plotted as white and blue dots, the bulk catalog of [20] is in gray. Subduction interface depth contours are displayed in black 41. The focal mechanism corresponds to an average mechanism computed from cataloged LFE detections [19]. (c.) shows the time-dip distribution of the events.

use this catalog as a starting point in our study. It should be noted that the events in the catalog are detected by a matched-filter search using template events, filtered between 1 and $2 \mathrm{~Hz}$. This band-pass is narrower than the range most studies of LFEs using matched-filter search have used up to now, usually $1-8 \mathrm{~Hz}[8,12, e . g]$

Nonetheless, we show in appendix A.1 that performing the matched-filter search in a narrow band does not bias the event detection and measurements of events' seismic moments and corner frequencies. Within the range of measured seismic moments, events with a corner frequency higher than $1 \mathrm{~Hz}$ do not have a lower probability of being detected because of the band-limited nature of detection.

\section{Selection of LFEs with high-quality waveforms}

The catalog of [20] has been created on the network-based template matching detection method [27, for instance]. The advantage of this method is that it detects many events with very weak signals hidden in the noise. At the same time, the signal-to-noise ratio for most LFEs in the final catalog is too low to be suited for a determination of their source parameters. Therefore, we selected LFEs with high-quality waveforms that could be used to robustly measure their seismic moments and corner frequencies.

Some single-component waveforms of catalog detections are contaminated with very high amplitude spikes, instrumental noise or waves from strong earthquakes. In these cases, useful signals are masked and corresponding waveforms cannot be used for determination of the LFE source parameters. We remove those seismograms from the analysis. Determination of seismic moment requires knowing the earthquake source location. However, LFE family locations determined by 20] contain a strong uncertainty on 
position in the direction perpendicular to the station array. This uncertainty is inherent to the linear observation geometry of the MASE network. Consequently, a few families of LFEs have templates with diffuse seismic phases, indicating that their measured position is loosely constrained. We therefore remove those families of events from our analysis.

We establish a set of criteria to automatically select LFEs and waveforms suitable for measuring the source parameters. We select $5 \mathrm{~s}$ long signals starting $2 \mathrm{~s}$ before the cataloged detection time of LFE S-waves.

This allows us to capture the most impulsive part of the S-wave signal, and allows for slight location and detection time imprecisions. This is done so as to trade-off between keeping a maximum of information and not allowing for too much diffuse seismic energy in the form of other seismic phases and events. By isolating the most impulsive part of the wave train, we best constrain the characteristic frequencies and seismic moment of the event.

In a next step, we reject signals with low amplitude and impulsivity to exclude false detections and contaminations with surface waves. First we define two frequency ranges in which we determine both an impulsivity $I$ and an amplitude $A$ : they will allow the criterion to be more flexible as for which events are taken into consideration. The impulsivity is calculated as the ratio of the seismic energy in the waveform time window $\mathcal{W}$ to the seismic energy contained in a three times larger background time window $\mathcal{B}$ around the detection, containing it:

$$
I=\frac{\int_{\mathcal{W}} s(t)^{2} d t}{\int_{\mathcal{B}} s(t)^{2} d t}
$$

where $s(t)$ is the velocity signal. $I$ varies between 0 , for least impulsive, and 1 , for most impulsive. It is used as a proxy of the detection SNR, but it also addresses the difficulty of defining what is noise within the tremor, where the LFE rate is very high. The amplitude is calculated as the maximum of the envelope of the filtered signal within the $5 \mathrm{~s}$ time window. For this purpose, the waveforms are converted into complex analytical signals $s^{A}(t)$ :

$$
\begin{aligned}
s^{A}(t) & =E(t) \cdot e^{i 2 \pi f^{i}(t)} \\
A & =\max _{\mathcal{W}}(E(t))
\end{aligned}
$$

$E(t)$ is the signal envelope and $f^{i}(t)$ is its instantaneous frequency. The latter will be used later on to determine the corner frequency of the selected LFEs.

Amplitudes and impulsivities are computed for all events on each channel of each station in the 1-2 $\mathrm{Hz}$ frequency band. This frequency range is characteristic of Mexican tremor signal in velocity, and has been shown to yield the best SNR for LFEs in this region [20, 51].

So as to robustly ensure that the detection is a correctly located LFE we also base the selection criterion on the correlation coefficient used in the making of the LFE catalog. It is a measure of the correlation of an event waveforms on all 15 detection channels to the stacked-waveforms template of its family 20. We consider the highest absolute values of the correlation coefficient to represent a wellconstrained location and an impulsive event. As a sum of 15 normalized correlation coefficients, it is normalized by the number of channels used in the detection of the event and thus varies between 0 and 1.

The selected waveforms have to fulfill the following criterion, where $I$ is the waveform impulsivity and $A$ its amplitude:

- The LFE should be impulsive, but waveforms with very high impulsivity are instrumental noise or earthquake wave arrivals:

$$
0.5<I<0.92
$$

- Higher-amplitude LFEs are generally detected in distant earthquakes waveforms. Therefore, we empirically limit the amplitude of a selected detection to $2 \times 10^{-6} \mathrm{~m} . \mathrm{s}^{-1}$. A lower threshold for selection $T_{\text {low }}(s t, c h)$ is fixed for each station and component (approximately $5 \times 10^{-9} \mathrm{~m} \cdot \mathrm{s}^{-1}$ ). Both bounding values are manually determined using the impulsivity-amplitude distribution on each channel of each station (see Figure 2).

$$
T_{\text {low }}(\text { st }, \text { ch })<A<2 \times 10^{-6} \text { m.s }{ }^{-1}
$$




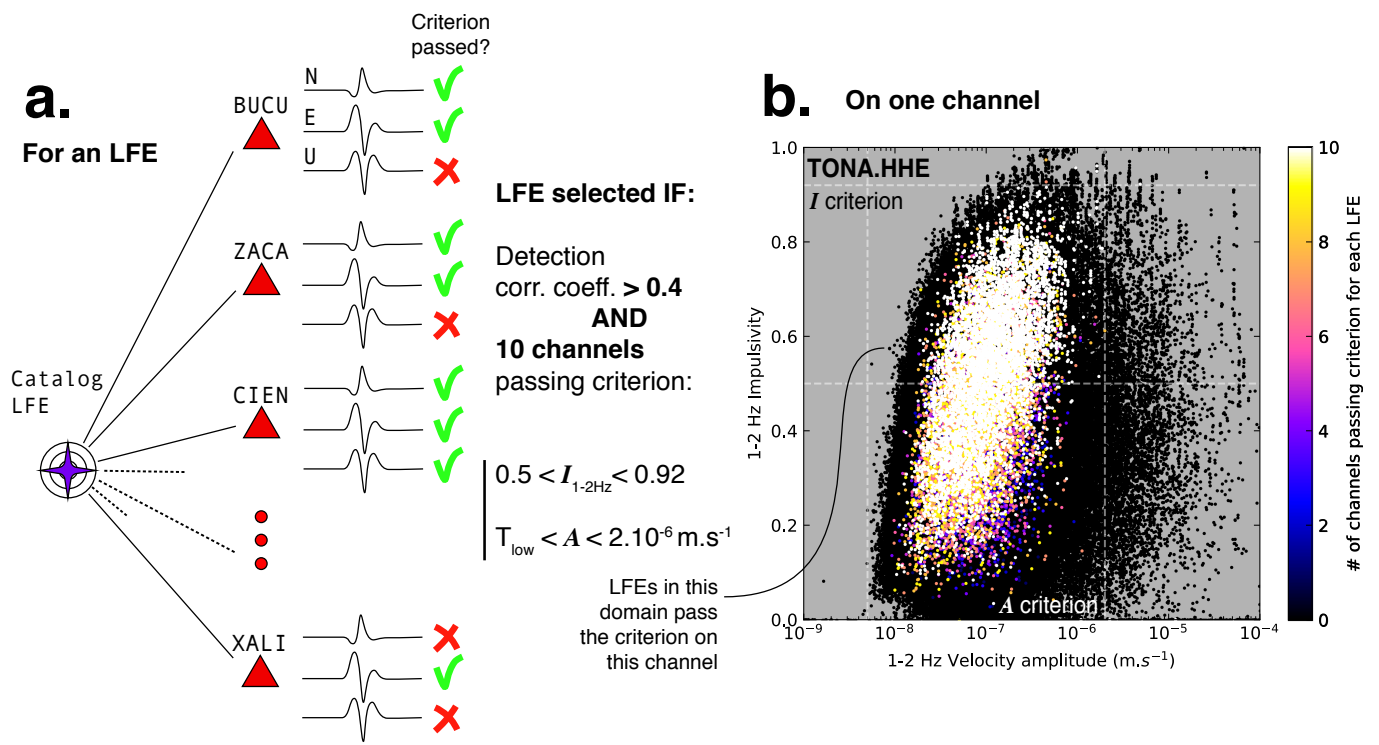

Figure 2: Selection of LFEs - (a.) Schematic recapitulation of the selection procedure. (b.) Amplitudeimpulsivity distribution of all detections recorded on the East component of the station TONA. Each dot is an LFE, its color corresponds to the number of channels on which it is recorded that pass the criteria. Selected LFEs are white dots: they are recorded on 10 or more channels passing the criterion and fulfill the recurrence and correlation requirements. The amplitude and first impulsivity criteria (equations 4 and 3) are shown with dashed lines. LFEs in the criterion domain pass the criterion on TONA.HHE, but 9 other records passing the criterion on other channels of the network are needed for an LFE to be selected.

For our source analysis, we select LFEs that contain at least 10 different channels satisfying the described criteria and have a network correlation coefficient superior to 0.4. This value has been chosen empirically, as the median of the correlation coefficients in the catalog.

A benefit of the network template matching method is that it allows to detect earthquakes with very close origin times, and thus with overlapping signals at some stations. Overlapping signals are not suitable for the source parameter analysis. Therefore, if several LFEs are detected within a $10 \mathrm{~s}$ time window, only the LFE with the highest correlation coefficient, and thus most reliable waveforms, is kept. Figure 2 sums up the selection process.

The selection yields 3498 LFEs representing 822 families out of a total of 1120 . Each event is recorded on 10 selected channels over the network and shown with blue circles in Figure 1. The most impulsive waveforms of two selected LFEs are displayed in Figure 3 . The selected LFEs exhibit a similar activity behavior in space and time as 20. observed for the whole catalog. First, they occur mainly in bursts within the sweet spot region. Second, during the 2006 SSE, bursts are no longer limited to the sweet spot, but extend updip in the transient zone. The activity in those two regions resembles the description [20], the sweet spot has a nearly constant activity over the 2-year span, whereas the transient zone is active mainly during the 2006 SSE and more sparse in the inter-SSE period.

\section{Characterizing seismic moment and corner frequency}

\subsection{Seismic moment}

We estimate seismic moment $M_{0}$ from the displacement spectra of S-waves $\widetilde{u}(f)$ that converges to a constant value at low frequencies [1]. This constant is proportional to $M_{0}[1]$. For every selected 

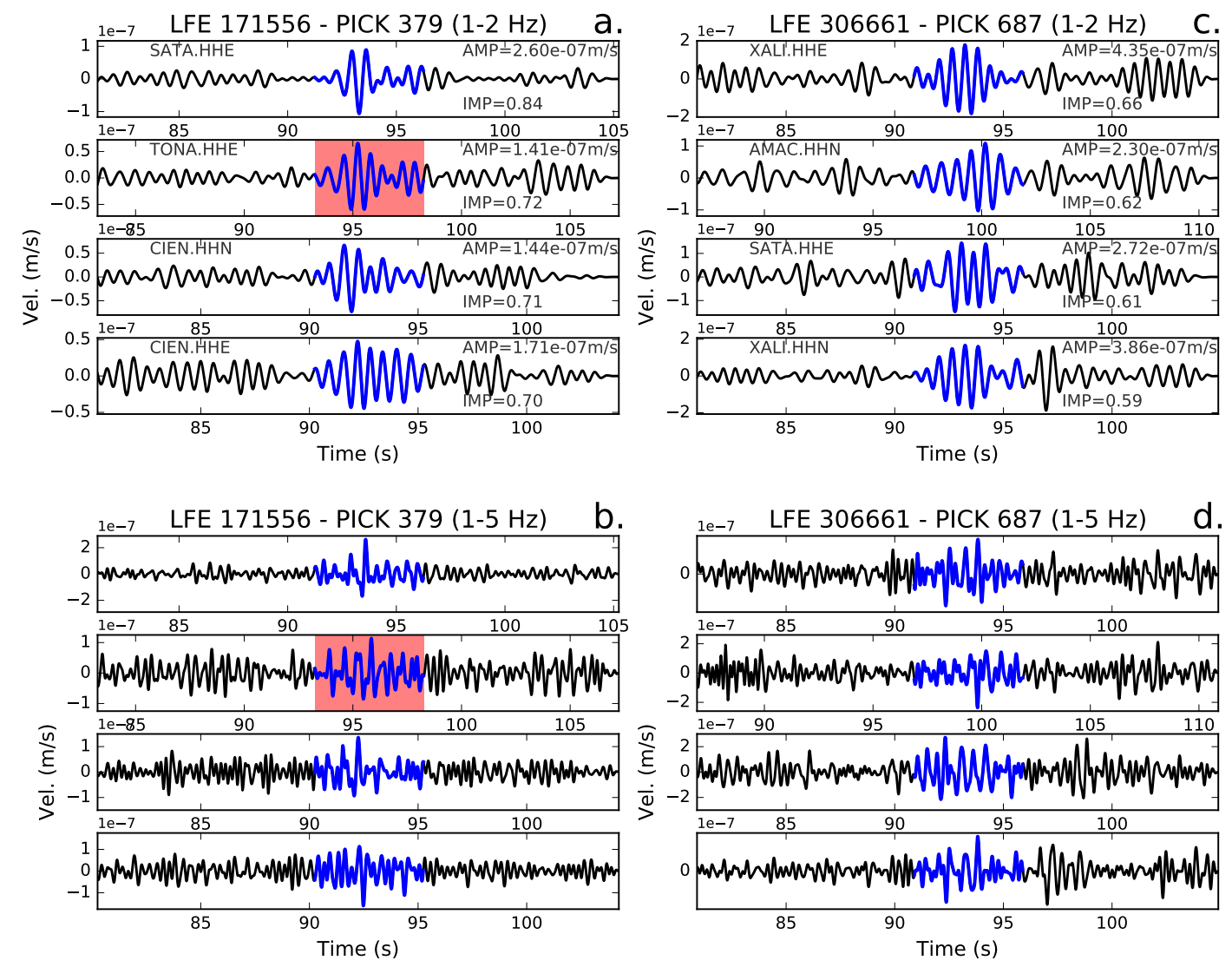

Figure 3: Selected LFEs' waveforms - The 4 most impulsive waveforms of LFE 379 and 687 are displayed here, 1-2 Hz band-pass filtered ((a.) and (c.)), and between 1 and $5 \mathrm{~Hz}((b$.$) and (d.)).$ The blue section of the waveform shows the time window used to define the detected signal, the red patch shows which channel's waveforms are used as an example for source parameters characterization in Figure 4. Impulsivity and amplitude are indicated for each channel.

waveform, we can determine the low-frequency spectral asymptotic value $\Omega_{0}{ }^{i, j}$ as:

$$
\Omega_{0}^{i, j}=\lim _{f \rightarrow 0} \widetilde{u}^{i, j}(f)=C^{i, j} \cdot M_{0}^{i, j},
$$

where $i$ corresponds to the LFE number and $j$ is an index given to each of the corresponding selected waveforms. The $C^{i, j}$ factor accounts for the source radiation pattern and the seismic wave propagation.

To measure these low-frequency asymptotic values, the $5 \mathrm{~s}$ long $\mathrm{S}$-wave velocity waveforms selected in the previous section are first detrended and high-pass filtered above $1 \mathrm{~Hz}$, to remove any influence of spectral leakage of microseismic noise, and then integrated into displacement. The power spectral density (PSD) of the displacement waveforms is computed with the multi-taper method [68]. It gives more robust spectral estimations than a simple discrete Fourier transform, especially for short signals. Taking the square root of this spectral estimation yields the displacement spectral amplitude. The lowfrequency value of the displacement spectral amplitude $\Omega_{0}{ }^{i, j}$ is estimated as the geometric mean of the 1-2 Hz portion of the spectral amplitude of displacement, filtered between 1 and $8 \mathrm{~Hz}$ (Equation 5 , and Figure 4 .

Then, seismic moment estimates from every waveform $M_{0}{ }^{i, j}$ can be retrieved by computing the propagation effects factor $C^{i, j}$. For this purpose, we compute synthetic waveforms for each sourcestation couple, using LFE family locations from [20] as hypocenters, an average regional seismic velocity model [36] and an average LFE focal mechanism, determined by [19]. We used the AxITRA software [14] based on the discrete wavenumber method [10].

After the synthetic seismograms are computed, we select a $5 \mathrm{~s}$ long time window around the S-waves, 

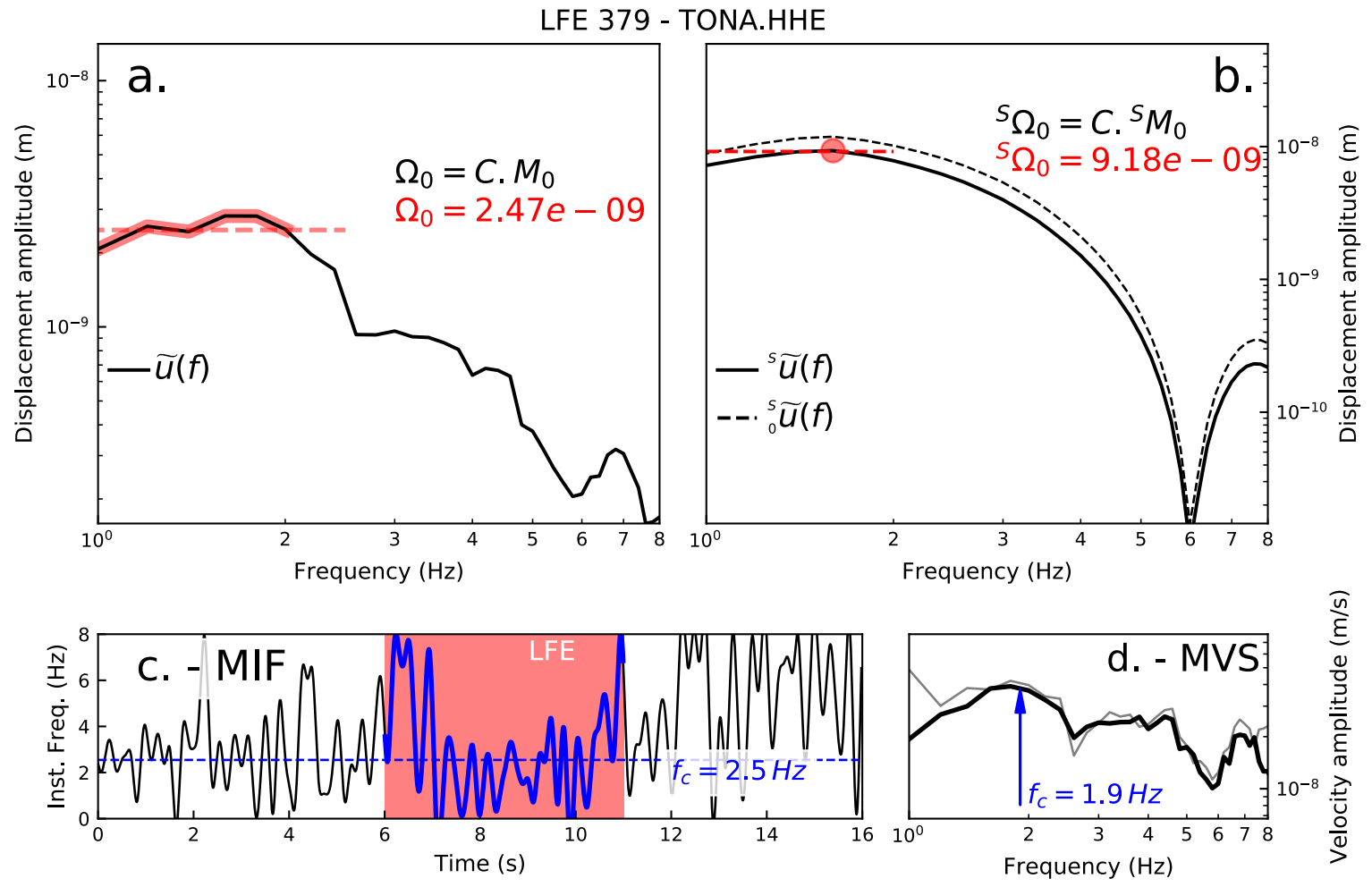

Figure 4: Determination of the seismic moment $M_{0}$ and corner frequency $f_{c}$, for an example lowfrequency earthquake - Example LFE: nb. 379, channel HHE of station TONA. (a.) $\Omega_{0}$ is determined as the red plateau of the displacement spectrum. (b.) ${ }^{s} \Omega_{0}$ is determined as the maximum of the synthetic displacement spectrum, taking into account attenuation. (c.) The corner frequency $f_{c}$ is measured as the mean instantaneous frequency (MIF) over the waveform time window (see Figure 3 for the LFE waveforms), (d.) and as the maximum of the velocity spectrum (MVS) of the waveform. In gray, the un-filtered velocity spectrum, allowing to assess the effects of the 1-8 $\mathrm{Hz}$ filter on the spectrum.

process them in the same way as the LFEs' seismogram and compute their displacement spectrum without attenuation ${ }_{0}^{s} \widetilde{u}(f)$, where $f$ is the frequency. The seismic wave attenuation effects are modeled in the spectral domain by introducing $a(f)$. A corrected synthetic spectral displacement ${ }^{s} \widetilde{u}(f)$ becomes:

$$
\begin{aligned}
{ }^{s} \widetilde{u}(f) & =a(f) \cdot{ }_{0}^{s} \widetilde{u}(f) \\
a^{i, j}(f) & =\exp \left(\frac{-2 \pi f X^{i, j}}{2 V_{S} Q(f)}\right), \text { where } Q(f)=273 f^{0.66}
\end{aligned}
$$

where the frequency-dependent quality factor $Q(f)$ for the Guerrero region has determined by [50], the average shear wave velocity $V_{S}$ is chosen to be $3.5 \mathrm{~km} . \mathrm{s}^{-1}$ and $X^{i, j}$ is the source-receiver distance.

We determine the low-frequency asymptote of the synthetic spectrum ${ }^{s} \Omega_{0}{ }^{i, j}$ as the maximum of the synthetic spectrum, filtered between 1 and $8 \mathrm{~Hz}$. Knowing the synthetic source moment ${ }^{s} M_{0}$ and following equation $(5), C^{i, j}$ can be eliminated:

$$
\frac{\Omega_{0}^{i, j}}{s \Omega_{0}{ }^{i, j}}=\frac{M_{0}^{i, j}}{{ }^{s} M_{0}},
$$

and a seismic moment estimated for each waveform of a selected LFE becomes:

$$
M_{0}{ }^{i, j}={ }^{s} M_{0} \frac{\Omega_{0}{ }^{i, j}}{s \Omega_{0}{ }^{i, j}} .
$$


Table 1: Measured LFE source parameters statistics analysis values

\begin{tabular}{|l|l|l|l|}
\hline Method $\backslash$ Frequencies (Hz) & Min. & Mean & Max. \\
\hline Instantaneous frequency & 2.0 & 3.18 & 5.52 \\
\hline Maximum of velocity sp. & 1.07 & 2.81 & 6.47 \\
\hline
\end{tabular}

\subsection{Corner frequency}

We use two different techniques to estimate the corner frequency from all selected waveforms for every LFE, $f_{c}{ }^{i, j}$. For both methods, the waveforms are detrended, corrected for attenuation (see equation 6 ) and band-pass filtered between 1 and $8 \mathrm{~Hz}$ to remove any influence of micro-seismic signal and higher frequency noise from the frequency band that correspond to LFEs frequencies. Further discussion on the choice of this frequency band can be found in the appendix, section A.2.

The first approach consists in automatically picking $f_{c}{ }^{i, j}$ as the frequency corresponding to the maximum in the velocity amplitude spectrum of every waveform. This method is labeled MVS (maximum of velocity spectrum) in the illustrations. The bandpass filter creates a slight amplitude reduction up to $1.5 \mathrm{~Hz}$, which can be seen when comparing the filtered and unfiltered spectra in Figure 4 This could shape the spectrum into a peak slightly above $1 \mathrm{~Hz}$, if the actual peak frequency between 1 and $8 \mathrm{~Hz}$ is close to $1 \mathrm{~Hz}$. However, we find that the $1-8 \mathrm{~Hz}$ filter band is the best compromise between allowing microseismic noise to bias the corner frequency measurement and slightly constraining the shape of the spectrum. The effect on measured corner frequencies of the low-cutoff of the filter is discussed more extensively in section A.2 and can be assessed in figure S2 of the supporting information file.

In the second approach, we estimate the corner frequency as the dominant instantaneous frequency of the S-waves. The waveforms are converted into complex analytical signals (see equation 2 ) and $f_{c}^{i, j}$ is estimated as the mean of the smoothed instantaneous frequency over the $5 \mathrm{~s}$ time window containing the S-waves. This method is labeled MIF (mean of instantaneous frequency) in the illustrations. For this method too, the effects of the filter band can be assessed in figure S2 of the supporting information file.

For each LFE, we obtain a final estimate of their moment $M_{0}{ }^{i}$ and two estimates of their corner frequency $f_{c}{ }^{i}$, all computed as medians of those measured from individual waveforms selected for this LFE. For each event, the uncertainty on its source parameters is estimated as the standard deviation of the values of the given source parameter measured on each record of the event.

\section{Results}

\subsection{Low-frequency earthquakes source parameters: seismic moment, corner frequency}

Seismic moments and corner frequencies estimated for the selected LFEs are shown in Figure 5 along with the schematic imprint of the $M_{0}-f_{c}$ distribution for classical earthquake, which follows $f_{c} \propto M_{0}{ }^{-\frac{1}{3}}$, for stress drops ranging from 0.1 to $100 \mathrm{MPa}$ [2], and values of $M_{0}$ and $f_{c}$ determined for LFEs in previous studies. Seismic moments and magnitudes estimated for Mexican LFEs range between $M_{0}=4.9 \times 10^{11}$ N.m $\left(M_{w} \sim 1.7\right)$ and $M_{0}=2.3 \times 10^{14}$ N.m $\left(M_{w} \sim 3.5\right)$. These values are higher than previous estimations in other regions 8 , 32. The median uncertainty on moment magnitude of $\delta M_{w} \approx 0.23$. It is mainly due to errors in modeling the velocity structure of the crust, assuming a generic radiation pattern for all events and large uncertainties on the hypocentral locations [20, supplementary figures 3 to 17]. The distribution of seismic moments above a threshold magnitude of $M_{w t h r} \sim 2.32$ follows a power-law, with an exponent $\beta=1.62$ (corresponding b-value $b=2.43$, see Appendix, Figure 7).

The measured corner frequencies mean and extrema for each method are displayed in Table 1 . They are approximately centered around $f_{c} \sim 3.0 \mathrm{~Hz}$ and fall within the accepted range of characteristic frequencies for LFEs of $1-6 \mathrm{~Hz}\left[8,32,65,67\right.$. The median uncertainty on corner frequency is $\delta f_{c} \approx 1.6$ for the maximum of velocity spectrum method, and $\delta f_{c} \approx 0.8$ for the mean instantaneous frequency method.

\subsection{Moment-duration scaling}

The $M_{0}-f_{c}$ distribution in Figures 5 and 6 shows that the corner frequencies seem virtually independent of seismic moment. In order to estimate the scaling law between the source parameters, $M_{0}-f_{c}$ points are 


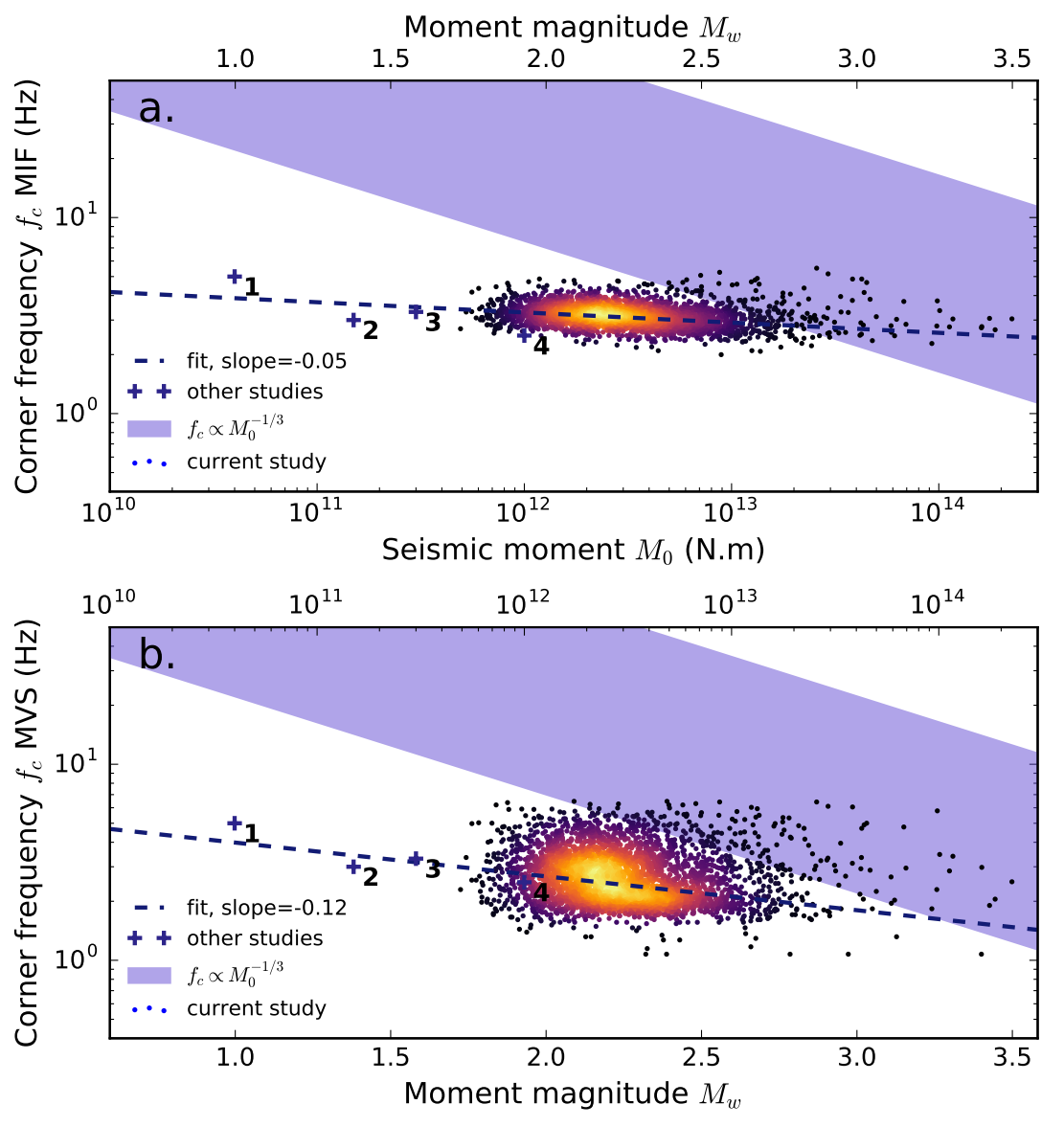

Figure 5: Distribution of the measured source parameters — The distribution is displayed and colored according to the point density for both methods of determining $f_{c}$ : (a.) with mean instantaneous frequency $(\mathrm{MIF}),(b$.$) as the frequency of the maximum of the velocity spectrum (MVS). Weighted$ least-square fits to the binned moments (see Figure 6) are shown as dotted lines, in each respective plot. Generic values for $M_{0}$ and $f_{c}$ measured in other regions are displayed along the distribution (1. 67, 2 . [66], 3. [32, 4. [8]) The self-similarity scaling law for classic earthquakes is represented by the purple patch. The higher bounding line corresponds to a stress drop of $100 \mathrm{MPa}$ and the lower to a stress drop of $0.1 \mathrm{MPa}$ [2, 16, 48.

binned into 35 bins of ascending moment magnitude, containing 100 points each. Moment magnitude of the bin is considered to be the mean $M_{w}$ of the events in the bin. Corner frequency of the bin $f_{c}^{b i n}$ is the weighted mean of the measured corner frequency of the binned events. The weight associated to the corner frequency of event $k$ is chosen to be the inverse of the variance of corner frequencies measured on each record for event $k, w_{k}=1 / \sigma_{k}^{2}$. Figure 6 shows the bin distribution and the associated estimation of variability of corner frequency within bin, calculated as a weighted standard deviation:

$$
\sigma^{b i n}=\sqrt{\frac{\sum_{k}\left(f_{c}{ }^{b i n}-f_{c}{ }^{k}\right)^{2} \times w_{k}}{\sum_{k} w_{k}}}
$$

with $f_{c}{ }^{k}$ the measured corner frequency for event of index $k$ in the considered bin, $w_{k}=1 / \sigma_{k}{ }^{2}$ its associated weight (see above), and $f_{c}^{\text {bin }}$ as the bin's corner frequency.

Using the bins $M_{0}$ and $f_{c}$ and their associated weight $w^{b i n}=1 / \sigma^{b i n}{ }^{2}$, a weighted least-square fit to the following power law is performed, in log-log space:

$$
f_{c} \propto M_{0}^{-\alpha} \Longleftrightarrow \log \left(f_{c}\right)=A-\alpha \log \left(M_{0}\right) .
$$

The resulting values of the slopes confirm that corner frequencies are weakly dependent on seismic moment : for the mean instantaneous frequency method (MIF) used to obtain $f_{c}: \alpha=0.052$, for the 

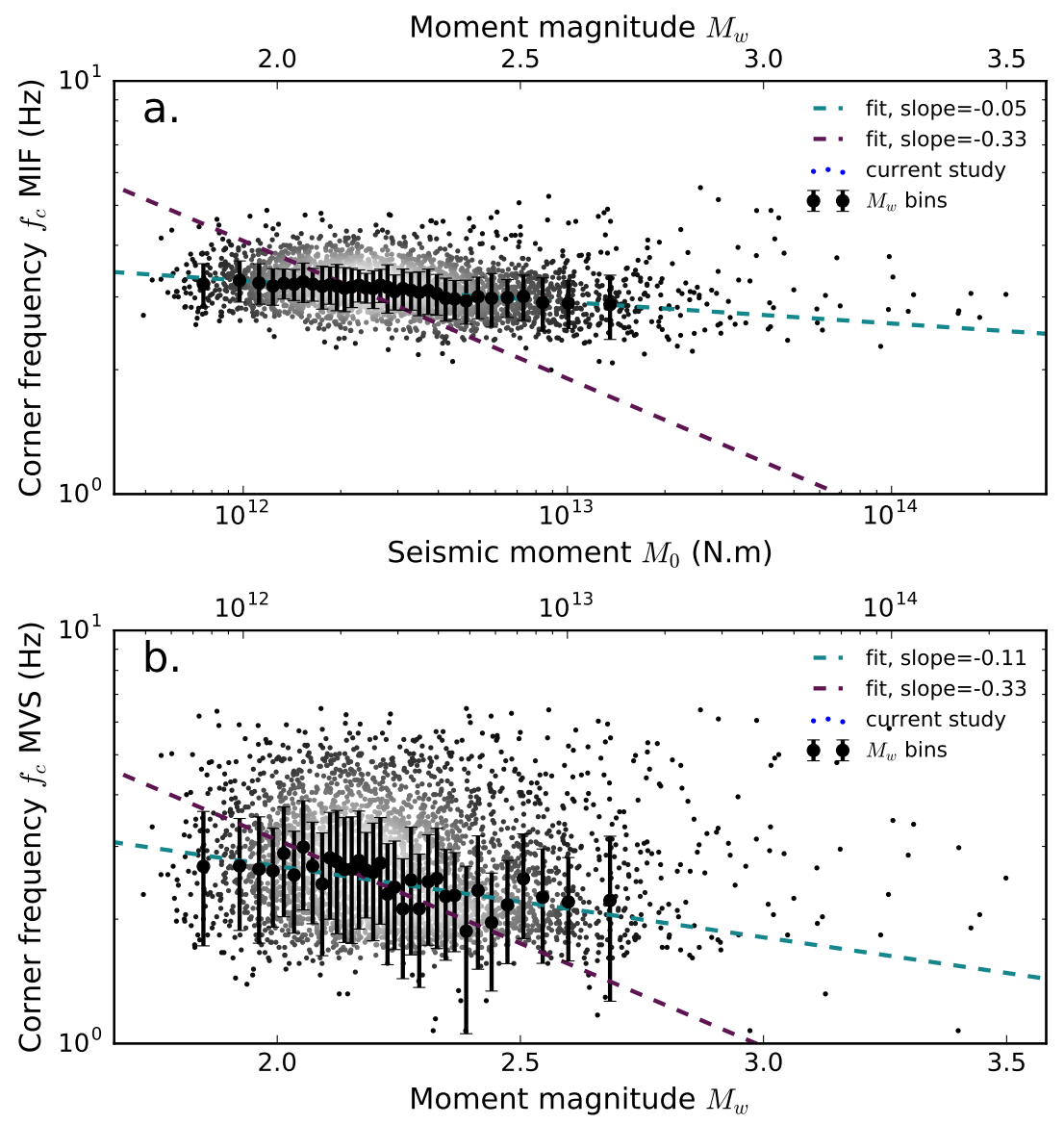

Figure 6: Binned distribution and uncertainties estimation - The measured $M_{0}-f_{c}$ is displayed and colored according to point density, for both the mean instantaneous frequency (MIF) and maximum of velocity spectrum (MVS). Seismic moment and corner frequency are binned, and the weighted standard deviation is shown with the error bars (see text for details). Weighted least-square fits to the binned data are displayed, the fit resulting in the purple scaling has $\alpha=\frac{1}{3}$ as a constraint.

maximum of velocity spectrum method (MVS): $\alpha=0.115$, (see Figures 5 and 6 ). This result is similar to the moment-duration scaling observed for LFEs in the Cascadia subduction zone by [8], where $\alpha \sim \frac{1}{10}$.

We proceed to estimate the probability density function (PDF) of the exponent of the scaling using a bootstrapping method. For each bin, we keep its moment but pick a new corner frequency from a normal distribution parametrized by the bin's corner frequency $\mu=f_{c}{ }^{b i n}$ and by the bin's weighted standard deviation $\sigma=\sigma^{\text {bin }}$ (see equation 9). An unweighted, least-square fit to a power law (equation 10 is then performed on the bin's seismic moment and re-sampled corner frequency. After 200,000 iterations of the previous steps, we obtain an estimate of the probability distribution function for the scaling parameter $\alpha$. The mean and standard variation of the obtained distribution give a new estimation of the slope and its associated uncertainty. For the MIF method, $\alpha=0.054 \pm 0.034$, for the MVS method, $\alpha=0.12 \pm 0.11$. Assuming that $\alpha$ is normally distributed, the probability that it is higher than 0.25 (thus closer to $\frac{1}{3}$ ) for our $f_{c}-M_{0}$ distribution is around 0.12 for the MVS method ( $\alpha=\frac{1}{3}$ is $\sim 1.2 \sigma$ away from the mean $\alpha$ ), and lower than $10^{-8}$ for the MIF method ( $\alpha=\frac{1}{3}$ is $\sim 5.7 \sigma$ away from the mean $\alpha$ ).

The scaling exponent $\alpha$ we measure is thus significantly lower than the earthquake power law exponent $\alpha=\frac{1}{3}$, for the MIF method at least. The MVS method gives more ambiguous results with regard to the value of the exponent $\alpha$, due to the wider distribution on $f_{c}$. 


\subsection{Stress-drop variations}

The stress-drop for an event of seismic moment $M_{0}$ modeled by a circular dislocation of radius $r$ is expressed as follows [4]:

$$
\Delta \sigma=\frac{7}{16} \frac{M_{0}}{r^{3}}
$$

Assuming a rupture velocity proportional to the shear wave velocity, $v_{r} \propto V_{S}$, it follows that the source radius is inversely proportional to the corner frequency [48]:

$$
r \propto \frac{V_{S}}{f_{c}} .
$$

From equations 11 and 12 and keeping the assumptions that our events originate from regions of constant $V_{S}$ and share the average corner frequency of our distribution $f_{c} \sim 3 \mathrm{~Hz}$, we can express the stress-drop ratio for two events of seismic moment $M_{01}$ and $M_{02}$ :

$$
\begin{aligned}
\Delta \sigma & \propto M_{0} \\
\Longrightarrow \frac{\Delta \sigma_{1}}{\Delta \sigma_{2}} & =\frac{M_{01}}{M_{02}}
\end{aligned}
$$

Using the extrema of the seismic moment distribution, and within the limit of our assumptions, we observe a stress-drop variation of a factor 300 across the range of measured seismic moments. For illustration purposes, we calculate what values would the stress-drop of our events take under the assumption that they have the same rupture speed as classical earthquakes, $v_{r}=0.9 V_{S}$. It yields $r=0.21 \frac{V_{S}}{f_{c}}$ [48, and using equation 11, the selected events have stress-drop ranging from $3 \mathrm{kPa}$ to $1 \mathrm{MPa}$, with most events around $17 \mathrm{kPa}\left(M_{0} \sim 2 \times 10^{12} \mathrm{~N} . \mathrm{m}\right)$. In the following section, we discuss a possible mechanism for the observed range of stress-drops.

\section{Discussion and interpretation of the observed scaling law}

\subsection{Scaling laws of earthquakes, slow earthquakes and LFEs}

The results of our analysis show that the selected LFEs follow a moment-duration scaling $f_{c} \propto M_{0}{ }^{-\alpha}$ with an exponent significantly lower than the value for regular earthquakes $\alpha=1 / 3$ [2, 29, 154, among others], around $\alpha \sim 0.1$ and $\alpha \sim 0.05$. The scaling found for selected LFEs in Guerrero concurs with the one found for LFEs in Cascadia by [8], who found a power law exponent of $\alpha=\frac{1}{10}$, quite similar to the scaling described in this paper. It is noteworthy that the detection method used by [8] relies on a matched-filter search, similarly to the catalog used in our study. However, we argue that performing a matched-filter search in the 1-2 Hz band sets a lower bound, but no upper bound for the characteristic frequency, for a given seismic moment. Therefore, the values of corner-frequencies should not be the product of the detection method, but reflect the real moment-corner frequency distribution of events, within the measured magnitude range. This argument is developed in appendix A.1. Interestingly enough, 66] found the moment-duration scaling for LFEs in Nankai tremor areas to be similar to regular earthquakes, $\alpha=\frac{1}{3}$. This discrepancy will not be thoroughly discussed here, but brings out intriguing questions, about the possibly different nature of the processes recorded in Guerrero, Cascadia and Nankai, and on the other hand, the potential detection and method biases that can lead to measuring different scalings.

Using a stochastic model describing a slow earthquake rupture (the Brownian slow earthquake model), [34, 35] showed that large-scale slow earthquakes as slow slip events' duration and seismic moment should follow a $T \propto M_{0}$ scaling, whereas on the scale of LFEs, slow earthquake events should follow a $T^{2} \propto M_{0}$ scaling, corresponding a value of $\alpha=1 / 2$. In [34, it is noted that, due to the fact that detection is only possible above noise levels, the apparent duration of LFEs should be shorter than expected, bringing them closer to a moment-duration scaling with $\alpha=1$. The LFEs we analyzed in Guerrero and the LFEs of Cascadia [8] cannot be described by either scaling laws.

There is evidence that the shortest slow slip transients measured to this day are self-similar, exhibiting a $T^{3} \propto M_{0}$ scaling law 23 . The exact proportionality measured for larger scales slow earthquakes 32 could be due to the fact that the largest ruptures are bounded in their growth by the rheological properties of the fault zone, whereas smaller events growth is un-bounded, and thus self-similar [28]. This geometric argument is valid both for slow and regular earthquakes, and further isolates LFEs momentduration characteristic values and scaling from the ones of slow and regular slip. For LFEs in Cascadia 
and Guerrero, a satisfactory physical description that could reproduce the observation of the constant duration and relatively low-frequency content still needs to be devised.

\subsection{Potential physical mechanisms accounting for magnitude-independent duration of LFEs}

\subsubsection{A source of constant size, but variable slip}

The scaling with $\alpha=\frac{1}{3}$ for regular earthquake is explained by the self-similarity of the seismic rupture. It develops with a constant ratio between the characteristic length of the rupture and fault slip, and produces a magnitude-independent stress drop 2, 38, 54. Events with a wide range of magnitudes but with nearly constant durations could thus be generated by variable amounts of slip on asperities of nearly constant sizes, producing a very limited range of recorded signal duration $\left(f_{c}\right)$ but variable seismic moments 8 , 45, 47. Independently from the current study, 23, found that, in Guerrero, the seismic moment rate, calculated from LFEs median amplitude during rapid slow slip transients scales with the moment rate calculated using GPS displacements during the same transient events. This reflects that the higher the seismic moment of LFEs, the higher the slip would be around the LFEs source. Combining those two observations leads to thinking of LFE sources in Guerrero as patches of fault of nearly constant area, that are forced to rupture by the surrounding slow, aseismic slip, with variable amplitudes of slip directly related to the surrounding fault slip. A similar model has been designed to explain the observation of a size-limitation for LFEs in Cascadia, describing the LFE family patch as a collection of subpatches randomly activated, totaling to an apparent source radius of about $300 \mathrm{~m}[13$.

\subsubsection{Potential effects of fluid circulation and high fluid pressures}

Early work on tectonic tremor and LFEs made the hypothesis that they are generated by fluids circulating in the vicinity of the fault zone $39,40,49$. More recent studies have strengthened the link between nonstationnary, metamorphic fluid flow and migrations of LFEs and slow slip activity, based on numerical models of pore-pressure diffusion [15] and on analysis of the large-scale, spatio-temporal behavior of LFE activity 21 .

A study by 45 reported that microseismic events occurring during water circulation tests display the same independence of corner frequency on seismic moment that we observe for our LFEs. As for the LFEs in this study, they find that stress drop varies on more than two orders of magnitude across the range of moments of their events. Likewise, 47] reports that microseismic events detected in Taiwan exhibit the same characteristics. Both studies suggest that these observations can be interpreted as a systematic control of the events' stress-drop and duration by dynamic fluid injection. Indeed, fluid injection can reduce the effective normal stress on asperities of the fault where the events occur. As fluid is injected or when aseismic slip is active, rapid, strong, heterogeneous increase of pore pressure in the fault can dramatically decrease the effective normal stress and thus the strength of the fault, triggering seismic slip on localized asperities. Under these conditions, [45] and 47] argue that the heterogeneity of fluid injection is likely to produce the wide range of stress-drops they observe. As noted by the previous papers, reduced effective normal stress have also been suggested as a cause of the low values of stress drop of VLFEs in Japan [37. In the light of the similarity of our observations and the ones presented in the discussed studies, dynamic triggering of LFEs by fluid injection could be a relevant mechanism to explain the LFEs' stress drop values.

In order to account for the observation of events' constant duration, 47. proposes that slip could only be maintained while the pore pressure is high enough to allow for seismic rupture, that is until the injection stops and the high pore pressure transient diffuses. The duration of the recorded seismic event would thus be controlled by the fluid transport properties in the fault zone. Those conditions of rapid, localized fluid injection are plausible within large active fault zones [61. In subduction zones, fluids released by the dehydration of hydrous minerals in the downgoing slab circulate within a permeable channel formed along or within the fault zone 3,70 . If the permeability or width of the channel has strong lateral heterogeneities, 61 suggests that strong, localized pore pressure transients can be formed. The strong, transient gradients of pore pressure can act as a source of seismic waves. The duration of such a source would thus be the characteristic time taken by a pore pressure transient to diffuse. 61 shows that as with such a mechanism, this duration is only governed by the transport properties of the fault zone and independent of the source magnitude. Although more precise observations of the source radiation pattern of LFEs and a substantial modeling effort are needed to confirm this hypothesis, it is consistent with our observations. 
Another lead to account for the apparent magnitude-independent, low-frequency content of LFEs is to interpret it as the consequence of specific, near-source propagation effects [9]. In this study the authors assume the existence of a narrow region with a very strong shear wave attenuation around LFEs source and suggest that the observed depletion in high frequencies can be related to this strong near-source attenuation of seismic waves. Based on accounts of high compressional to shear wave velocity ratio $V_{P} / V_{S}$ in seismic cross-section of subduction zones [7, 63, tidal triggering of LFE activity [5, 58, 59, e.g.] and dynamic triggering by teleseismic waves [60, the source region of LFEs is thought to be under nearly lithostatic fluid pressures. Laboratory studies indicate that such high fluid content can also be the cause of strong shear wave attenuation [69].

\section{Conclusions}

We analyzed a subset of carefully selected LFEs from the cataloged events of Guerrero, Mexico [20]. The subset of LFEs samples a relatively broad range of seismic moments, between $M_{w} 1.7$ and $M_{w} 3$, with corner-frequencies clustering around $3 \mathrm{~Hz}$, between 1.5 and $6 \mathrm{~Hz}$. Overall, we find corner frequences to be very weakly dependent on seismic moments for LFEs in Guerrero. When trying to describe this relationship with the power law $f_{c} \propto M_{0}{ }^{-\alpha}$, we retrieve $\alpha=0.12 \pm 0.11$ and $\alpha=0.054 \pm 0.034$ for the two sets of corner frequency measurements. In both cases those scalings are significantly different from a self-similar rupture, for which $\alpha=1 / 3$.

Recent studies show that the clear departure from self-similarity measured for LFEs in Guerrero and Cascadia is not a necessary feature of LFEs in all tremor regions across the world. Exploring this regional specificity might bring significant insight on LFEs source physics and lead us to understand more about how deep fault environment affects slow slip processes.

Acknowledgements Data and materials availability: The source parameters data we generate in this study is available on the Slow Earthquake Database (http://www-solid.eps.s.u-tokyo.ac.jp/ sloweq/). The complete low-frequency earthquake catalog compiled by 20] can be found on the same archive. The MASE seismic dataset (https://doi.org/10.7909/C3RN35SP) used in this study is openly available from IRIS data center under the network code TO. Software use: One figure was made with the Generic Mapping Tools (GMT) [72]. We used the Python library ObsPy [44 for seismic data handling and the MTSPEC library [55] for multitaper spectral analysis. Research funding and support: This study was supported by the Russian Ministry of Education and Science (grant N 14.W03.31.0033) and by the European Research Council under the European Union Horizon 2020 research and innovation program (grant agreement no. 787399 - SEISMAZE). Numerical computations were performed on the S-CAPAD platform, at Institut ie Physique du Globe de Paris (IPGP), France. The authors confirm that there are no real or perceived conflicts of interest associated with this work. 


\section{A Effects of bandpass filtering on events corner frequencies}

\section{A.1 Detection bandpass filter}

The LFEs of the catalog used in this study were detected using a matched-filter search approach, with template waveforms band-pass filtered between $1-2 \mathrm{~Hz}[20$.

A potential concern is that a matched-filter search using a narrow band-pass would only identify events with a characteristic frequency between 1 and $2 \mathrm{~Hz}$. Assuming it was the case, it would bias our analysis towards the observed magnitude-independent corner frequencies.

However, the use of matched-filter search does not constrain the characteristic frequencies of the event between 1-2 Hz, it simply limits detections to signals with energy within this frequency band. Several studies using matched-filter search to detect classic earthquakes are able to detect events with a range of magnitudes, that, through the earthquake moment-duration scaling law, corresponds to a range of characteristic frequency wider than the chosen band-pass [71, figure 7.(b)], 22, figure 5]. In our case most events indeed have corner frequencies above $2 \mathrm{~Hz}$ and up to $6.5 \mathrm{~Hz}$ (see Table 1. Figure 5).

A compact form of the Brune spectrum for ground motion velocity can be used to compare the detectability of two events: $\widetilde{v}(f)$ :

$$
\widetilde{v}(f)=\frac{2 \pi C \cdot M_{0} \cdot f}{1+\left(\frac{f}{f_{c}}\right)^{2}},
$$

where $C$ is a constant relating to the velocity structure of the medium and the source focal mechanism, $M_{0}$ the seismic moment of the event and $f_{c}$ its corner frequency. For any two events with $f_{c 1}<f_{c 2}$ but identical moment, for any given frequency of detection $f$, the corresponding velocity amplitudes will follow $\widetilde{v_{1}}(f)<\widetilde{v_{2}}(f)$. In other words, for a given magnitude, events with a higher characteristic frequency than events we detected do not have a lower probability to be detected because of the band-pass filter. Therefore, within the range of moments that we measured, the detection band-pass filter should not bias corner frequencies by constraining them at low values, close to $2 \mathrm{~Hz}$. Using the same demonstration, events of characteristic frequency below $1 \mathrm{~Hz}$ and seismic moment within the range of moments we measure have a low probability to be detected by the matched-filter search. The matched-filter search band-pass thus imposes a lower bound on detections' corner frequencies in the range of moments we measure, but no higher bound. For events above $8 \mathrm{~Hz}$ however, the presence of high-frequency noise would bias the measure of corner frequencies, motivating our choice to limit our analysis to the $1-8 \mathrm{~Hz}$ frequency band.

As a conclusion, in the seismic moment range of our events $\left(8 \times 10^{11}-1 \times 10^{13} \mathrm{~N} . \mathrm{m}\right)$, the measure of corner frequency is not biased by the detection process, for values of corner frequency between 1 and $8 \mathrm{~Hz}$. A frequency range of around 1-10 Hz and range of moments 1.5 orders of magnitude wide were enough for [66] to measure a steeper dependence of corner-frequency on seismic moment, and should thus be enough in this study to estimate a moment-duration scaling without significant artifacts.

\section{A.2 Analysis bandpass filter}

In Figure S1, we see that the tremor seismic energy seems really bounded between $0.8 \mathrm{~Hz}$ and $8 \mathrm{~Hz}$. Above this band, high-frequency noise, possibly anthropogenic, is active, below this band, the seismic time-series are dominated by low-frequency noise leaking from microseismic modes. The choice of the 1-8 Hz band-pass filter used to measure corner-frequencies is motivated by this observation.

It is possible that corner frequencies of the event we detected are lower than $1 \mathrm{~Hz}$, and that the 1-8 $\mathrm{Hz}$ band-pass filter we use biases our measure of the corner frequencies to constrain them in a narrow band above $1 \mathrm{~Hz}$. We tested the influence of the lower cutoff frequency of the band-pass filter on the measured corner frequency. Results of this test are shown in Figure S2.

The distribution of corner frequencies is not drastically modified down to a lower cutoff of $0.2 \mathrm{~Hz}$, where corner frequencies suddenly regroup around $0.2 \mathrm{~Hz}$. This sudden shift is due to the filter band not blocking pollution of micro-seismic noise anymore.

In addition to it, corner frequencies are slightly dragged to lower values, as the lower cutoff decreases from $1 \mathrm{~Hz}$ to $0.5 \mathrm{~Hz}$. This should be expected: more and more pollution from micro-seismic noise is allowed in records of events on stations that witness higher amplitudes of micro-seismic noise, due to proximity to the ocean or site effects. On those records, the characteristic frequency will be picked lower, due to the influence of micro-seismic noise. Figure 4 (d.) is a good illustration of this: the peak of the un-filtered spectrum is located at $1 \mathrm{~Hz}$ due to micro-seismic noise influence. 
The $1-8 \mathrm{~Hz}$ is thus chosen as a compromise. The lower cutoff is taken far enough from micro-seismic frequencies so that the event signal is not polluted, even at stations where micro-seismic noise has the highest amplitude. Ultimately, it does constrain the corner frequency between 1 and $8 \mathrm{~Hz}$, but this seems reasonably wide.

\section{B Moment-frequency distribution of LFEs}

In Figure 7, we represent the complementary cumulative distribution function (abbreviated as CCDF, also called survival function) of seismic moments, $P\left(M_{0}>M_{0}{ }^{\prime}\right)$. It is computed with $M_{0}{ }^{\prime}$ regularly spaced in the magnitude range.

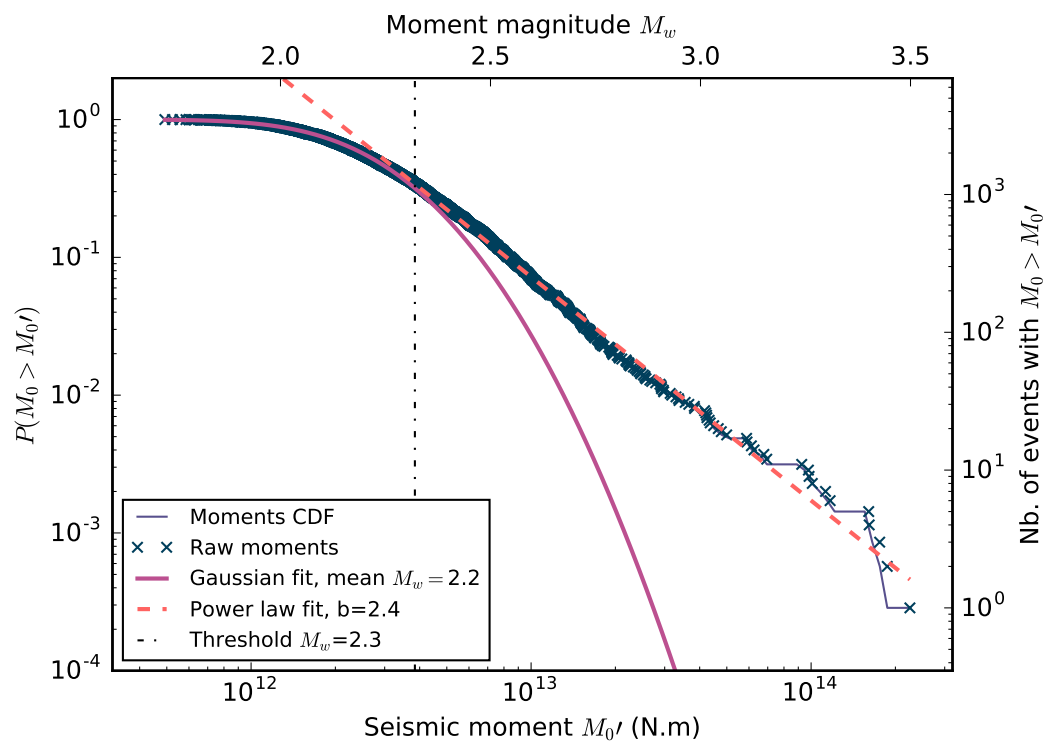

Figure 7: Moment-frequency distribution of the selected LFEs - The seismic moment complementary cumulative distribution function (CCDF) of our LFEs is modeled by a Gaussian below $M_{w t h r} 2.32$, with mean magnitude $M_{w} 2.4$, and by a power law above $M_{w t h r}$, with an exponent corresponding to a b-value of 2.4 .

The higher-moments tail of the distribution can be described as a power-law distribution, with the following probability density function (PDF) and CCDF:

$$
\begin{aligned}
p\left(M_{0}\right) & =\frac{\beta}{M_{0 t h r}}\left(\frac{M_{0}}{M_{0 t h r}}\right)^{-\beta-1} \\
P\left(M_{0}>M_{0}{ }^{\prime}\right) & =\left(\frac{M_{0}{ }^{\prime}}{M_{0 t h r}}\right)^{-\beta}
\end{aligned}
$$

where $M_{0 t h r}$ is the lower bound for which the power-law distribution is valid. The power law exponent $\beta$ relates to the b-value of the Gutenberg-Richter law following: $b=\frac{3}{2} \beta$. For moments above a threshold $M_{0 t h r}$, we perform an un-weighted, least-square fit of the logarithm of the moments CCDF to a powerlaw. We find that $\beta=1.62$, and a corresponding $b$-value of $b=2.43$. For comparison, $b$-values of classical seismicity are usually around 1 . The threshold magnitude $M_{w t h r}=2.32$ (corresponding to $M_{0 t h r}$ ) is selected so as to minimize the Kolmogorov-Smirnov statistic for the moments' distribution and the fitted power law.

The deviation from a power law at lower moments is often attributed to observational limitations: below a completeness magnitude, events are too weak to all be detected, and it manifests as a deviation from the otherwise assumed power law distribution of moments. Here, the completeness magnitude would thus be $M_{w t h r}=2.32$. The departure from a power-law at lower moments can also be the manifestation of a characteristic scale of magnitude of the events. Several studies report observations of LFEs size distribution being best modeled by exponential laws and thus of a characteristic scale controlling the LFE size distribution [12, 13. In our case, a normal distribution can be fitted to the distribution of moments below $M_{w t h r}$, parametrized by a mean magnitude $\overline{M_{w}}=\mu=2.23$ and a standard deviation 
$\sigma=0.19$. The mean magnitude $\overline{M_{w}}=2.23$ defines a characteristic scale of the lower-bound of the moment distribution. It is either due to a scale limitation of LFEs in Guerrero, or a manifestation of the limitation of events' detection. 


\section{References}

[1] K. Aki and P. G. Richards. volume 1, chapter 4.3, 10.1, pages 79-82, 497-498. University Science Books, 2002.

[2] B. P. Allmann and P. M. Shearer. Global variations of stress drop for moderate to large earthquakes. Journal of Geophysical Research: Solid Earth, 114(B1), 2009.

[3] S. Angiboust, S. Wolf, E. Burov, P. Agard, and P. Yamato. Effect of fluid circulation on subduction interface tectonic processes: Insights from thermo-mechanical numerical modelling. Earth Planet. Sci. Lett., 357-358:238-248, 2012. ISSN 0012-821X. doi:https://doi.org/10.1016/j.epsl.2012. 09.012. URL http://www.sciencedirect.com/science/article/pii/S0012821X12005080.

[4] Pascal Audet, Michael G. Bostock, Nikolas I. Christensen, and Simon M. Peacock. Seismic evidence for overpressured subducted oceanic crust and megathrust fault sealing. Nature, 457:76-78, 012009. URL http://dx.doi .org/10.1038/nature07650.

[5] Nicholas M Beeler, Amanda Thomas, Roland Bürgmann, and David Shelly. Inferring fault rheology from low-frequency earthquakes on the san andreas. Journal of Geophysical Research: Solid Earth, 118(11):5976-5990, 2013.

[6] Gregory C. Beroza and Satoshi Ide. Slow earthquakes and nonvolcanic tremor. Annual Review of Earth and Planetary Sciences, 39(1):271-296, 2011. doi:10.1146/annurev-earth-040809-152531. https://doi.org/10.1146/annurev-earth-040809-152531. URL https://doi.org/10.1146/ annurev-earth-040809-152531

[7] M. G. Bostock, A. A. Royer, E. H. Hearn, and S. M. Peacock. Low frequency earthquakes below southern vancouver island. Geochemistry, Geophysics, Geosystems, 13(11), 2012.

[8] M. G. Bostock, A. M. Thomas, G. Savard, L. Chuang, and A. M. Rubin. Magnitudes and momentduration scaling of low-frequency earthquakes beneath southern vancouver island. Journal of Geophysical Research: Solid Earth, 120(9):6329-6350, 2015.

[9] MG Bostock, AM Thomas, AM Rubin, and NI Christensen. On corner frequencies, attenuation, and low-frequency earthquakes. Journal of Geophysical Research: Solid Earth, 122(1):543-557, 2017.

[10] M. Bouchon. A review of the discrete wavenumber method. In Seismic Motion, Lithospheric Structures, Earthquake and Volcanic Sources: The Keiiti Aki Volume, pages 445-465. Springer, 2003.

[11] J. N. Brune. Tectonic stress and the spectra of seismic shear waves from earthquakes. Journal of geophysical research, 75(26):4997-5009, 1970. doi:10.1029/JB075i026p04997.

[12] C. J. Chamberlain, D. R. Shelly, J. Townend, and T. A. Stern. Low-frequency earthquakes reveal punctuated slow slip on the deep extent of the alpine fault, new zealand. Geochemistry, Geophysics, Geosystems, 15(7):2984-2999, 2014.

[13] SR Chestler and KC Creager. Evidence for a scale-limited low-frequency earthquake source process. Journal of Geophysical Research: Solid Earth, 122(4):3099-3114, 2017.

[14] O. Coutant. axitra software, 2008. https://isterre.fr/annuaire/pages-web-du-personnel/oliviercoutant/article/logiciels-softwares?lang $=$ fr.

[15] Víctor M Cruz-Atienza, Carlos Villafuerte, and Harsha S Bhat. Rapid tremor migration and porepressure waves in subduction zones. Nature communications, 9(1):2900, 2018.

[16] John Douglas Eshelby. The determination of the elastic field of an ellipsoidal inclusion, and related problems. Proceedings of the Royal Society of London. Series A. Mathematical and Physical Sciences, 241(1226):376-396, 1957.

[17] W. B. Frank. Slow slip hidden in the noise: The intermittence of tectonic release. Geophysical Research Letters, 43(19), 2016.

[18] W. B. Frank and N. M. Shapiro. Automatic detection of low-frequency earthquakes (lfes) based on a beamformed network response. Geophysical Journal International, 197(2):1215-1223, 2014. 
[19] W. B. Frank, N. M. Shapiro, V. Kostoglodov, A. L. Husker, M. Campillo, J. S. Payero, and G. A. Prieto. Low-frequency earthquakes in the mexican sweet spot. Geophysical Research Letters, 40 (11):2661-2666, 2013.

[20] W. B. Frank, N. M. Shapiro, A. L. Husker, V. Kostoglodov, A. Romanenko, and M. Campillo. Using systematically characterized low-frequency earthquakes as a fault probe in guerrero, mexico. Journal of Geophysical Research: Solid Earth, 119(10):7686-7700, 2014.

[21] W. B.. Frank, N. M. Shapiro, A. L. Husker, V. Kostoglodov, H. S. Bhat, and M. Campillo. Alongfault pore-pressure evolution during a slow-slip event in guerrero, mexico. Earth and Planetary Science Letters, 413:135-143, 2015.

[22] William B. Frank and Rachel E. Abercrombie. Adapting the Matched-Filter Search to a WideAperture Network: An Aftershock Sequence and an Earthquake Swarm in Connecticut. Bulletin of the Seismological Society of America, 108(1):524-532, February 2018. ISSN 0037-1106, 1943-3573. doi:10.1785/0120170190. URL https://pubs.geoscienceworld.org/ssa/bssa/article/108/ 1/524/525666/Adapting-the-MatchedFilter-Search-to-a

[23] William B Frank and Emily E Brodsky. Daily measurement of slow slip from low-frequency earthquakes is consistent with ordinary earthquake scaling. Science Advances, 5(10):eaaw9386, 2019.

[24] William B Frank, Mathilde Radiguet, Baptiste Rousset, Nikolaï M Shapiro, Allen L Husker, Vladimir Kostoglodov, Nathalie Cotte, and Michel Campillo. Uncovering the geodetic signature of silent slip through repeating earthquakes. Geophysical Research Letters, 42(8):2774-2779, 2015.

[25] William B Frank, Baptiste Rousset, Cécile Lasserre, and Michel Campillo. Revealing the cluster of slow transients behind a large slow slip event. Science advances, 4(5):eaat0661, 2018.

[26] A. Ghosh, J. E. Vidale, J. R. Sweet, K. C. Creager, A. G. Wech, H. Houston, and E. E. Brodsky. Rapid, continuous streaking of tremor in cascadia. Geochemistry, Geophysics, Geosystems, 11(12), 2010.

[27] Steven J. Gibbons and Frode Ringdal. The detection of low magnitude seismic events using array-based waveform correlation. Geophysical Journal International, 165(1):149-166, 2019/02/05 2006. doi:10.1111/j.1365-246X.2006.02865.x. URL https://doi.org/10.1111/ j.1365-246X.2006.02865.x.

[28] Joan Gomberg, Aaron Wech, Kenneth Creager, Kazushige Obara, and Duncan Agnew. Reconsidering earthquake scaling. Geophysical Research Letters, 43(12):6243-6251, 2016.

[29] Heidi Houston. Influence of depth, focal mechanism, and tectonic setting on the shape and duration of earthquake source time functions. Journal of Geophysical Research: Solid Earth, 106(B6): 11137-11150, 2019/02/03 2001. doi:10.1029/2000JB900468. URL https://doi.org/10.1029/ 2000JB900468.

[30] Heidi Houston, Brent G Delbridge, Aaron G Wech, and Kenneth C Creager. Rapid tremor reversals in cascadia generated by a weakened plate interface. Nature Geoscience, 4(6):404, 2011.

[31] A. Husker, I. Stubailo, M. Lukac, V.a Naik, R. Guy, P. Davis, and D. Estrin. Wilson: The wirelessly linked seismological network and its application in the middle american subduction experiment. Seismol. Res. Lett., 79:438-443, 2008.

[32] S. Ide, G. C. Beroza, D. R. Shelly, and T. Uchide. A scaling law for slow earthquakes. Nature, 447 (7140):76-79, 2007.

[33] S. Ide, D. R. Shelly, and G. C. Beroza. Mechanism of deep low frequency earthquakes: Further evidence that deep non-volcanic tremor is generated by shear slip on the plate interface. Geophysical Research Letters, 34(3), 2007.

[34] Satoshi Ide. A brownian walk model for slow earthquakes. Geophysical Research Letters, 35(17), 2008.

[35] Satoshi Ide and Julie Maury. Seismic moment, seismic energy, and source duration of slow earthquakes: Application of brownian slow earthquake model to three major subduction zones. Geophysical Research Letters, 45(7):3059-3067, 2018. 
[36] A. Iglesias, R. W. Clayton, X. Pérez-Campos, S.K. Singh, J. F. Pacheco, D. García, and C. ValdésGonzález. S wave velocity structure below central mexico using high-resolution surface wave tomography. Journal of Geophysical Research: Solid Earth, 115(B6), 2010.

[37] Yoshihiro Ito and Kazushige Obara. Very low frequency earthquakes within accretionary prisms are very low stress-drop earthquakes. Geophysical Research Letters, 33(9), 2006.

[38] Hiroo Kanamori and Don L. Anderson. Theoretical basis of some empirical relations in seismology. Bulletin of the Seismological Society of America, 65(5):1073-1095, 1975.

[39] Honn Kao, Shao-Ju Shan, Herb Dragert, Garry Rogers, John F. Cassidy, and Kumar Ramachandran. A wide depth distribution of seismic tremors along the northern cascadia margin. Nature, 436:841844, 08 2005. doi:10.1038/nature03903. URL http://dx.doi.org/10.1038/nature03903

[40] A. Katsumata and N. Kamaya. Low-frequency continuous tremor around the moho discontinuity away from volcanoes in the southwest japan. Geophysical Research Letters, 30(1), 2003.

[41] Y. Kim, R. W. Clayton, and J. M. Jackson. Geometry and seismic properties of the subducting cocos plate in central mexico. Journal of Geophysical Research: Solid Earth, 115(B6), 2010.

[42] V. Kostoglodov and J. F. Pacheco. Cien años de sismicidad en méxico, instituto de geofísica, universidad nacional autónoma de méxico, 1999. URL http://usuarios.geofisica.unam.mx/ vladimir/sismos/100years.html.

[43] V. Kostoglodov, A. Husker, N. M Shapiro, J. S. Payero, M. Campillo, N. Cotte, and R. Clayton. The 2006 slow slip event and nonvolcanic tremor in the mexican subduction zone. Geophysical Research Letters, 37(24), 2010.

[44] L. Krischer, T. Megies, R. Barsch, M. Beyreuther, T. Lecocq, C. Caudron, and J. Wassermann. Obspy: A bridge for seismology into the scientific python ecosystem. Computational Science \& Discovery, 8(1):014003, 2015.

[45] Olivier Lengliné, L Lamourette, L Vivin, Nicolas Cuenot, and Jean Schmittbuhl. Fluid-induced earthquakes with variable stress drop. Journal of Geophysical Research: Solid Earth, 119(12):89008913, 2014.

[46] Olivier Lengliné, WB Frank, D Marsan, and J-P Ampuero. Imbricated slip rate processes during slow slip transients imaged by low-frequency earthquakes. Earth and Planetary Science Letters, 476: 122-131, 2017.

[47] Yen-Yu Lin, Kuo-Fong Ma, Hiroo Kanamori, Teh-Ru Alex Song, Nadia Lapusta, and Victor C Tsai. Evidence for non-self-similarity of microearthquakes recorded at a taiwan borehole seismometer array. Geophysical Journal International, 206(2):757-773, 2016.

[48] Raul Madariaga. Dynamics of an expanding circular fault. Bulletin of the Seismological Society of America, 66(3):639-666, 1976.

[49] K. Obara. Nonvolcanic deep tremor associated with subduction in southwest japan. Science, 296 (5573):1679-1681, 2002.

[50] Mario Ordaz and Shri Krishna Singh. Source spectra and spectral attenuation of seismic waves from mexican earthquakes, and evidence of amplification in the hill zone of mexico city. Bulletin of the Seismological Society of America, 82(1):24-43, 1992.

[51] J. S. Payero, V. Kostoglodov, N. Shapiro, T. Mikumo, A. Iglesias, X. Pérez-Campos, and R. W. Clayton. Nonvolcanic tremor observed in the mexican subduction zone. Geophysical Research Letters, 35(7), 2008.

[52] X. Perez-Campos, Y. Kim, A. Husker, P. Davis, R. Clayton, A. Iglesias, J. F. Pacheco, S. K. Singh, V. C. Manea, and M. Gurnis. Horizontal subduction and truncation of the cocos plate beneath central mexico. Geophys. Res. Lett., 35:L18303, 2008. doi:10.1029/2008GL035127. 
[53] Natalia Poiata, Jean-Pierre Vilotte, Pascal Bernard, Claudio Satriano, and Kazushige Obara. Imaging different components of a tectonic tremor sequence in southwestern japan using an automatic statistical detection and location method. Geophysical Journal International, 213(3):2193-2213, 2018.

[54] G. A. Prieto, P. M. Shearer, F. L. Vernon, and D. Kilb. Earthquake source scaling and self-similarity estimation from stacking p and s spectra. Journal of Geophysical Research: Solid Earth, 109(B8), 2004.

[55] G. A. Prieto, R. L. Parker, and F. L. Vernon III. A fortran 90 library for multitaper spectrum analysis. Computers $\&$ Geosciences, 35(8):1701-1710, 2009.

[56] M. Radiguet, F. Cotton, M. Vergnolle, M. Campillo, A. Walpersdorf, N. Cotte, and V. Kostoglodov. Slow slip events and strain accumulation in the guerrero gap, mexico. Journal of Geophysical Research: Solid Earth, 117(B4), 2012.

[57] G. Rogers and H. Dragert. Episodic tremor and slip on the cascadia subduction zone: The chatter of silent slip. Science, 300(5627):1942-1943, 2003.

[58] A. A. Royer, A. M. Thomas, and M. G. Bostock. Tidal modulation and triggering of low-frequency earthquakes in northern cascadia. Journal of Geophysical Research: Solid Earth, 120(1):384-405, 2015 .

[59] J. L. Rubinstein, M. La Rocca, J. E. Vidale, K. C. Creager, and A. G. Wech. Tidal modulation of nonvolcanic tremor. Science, 319(5860):186-189, 2008.

[60] J. L. Rubinstein, J. Gomberg, J. E. Vidale, A. G. Wech, H. Kao, K. C. Creager, and G. Rogers. Seismic wave triggering of nonvolcanic tremor, episodic tremor and slip, and earthquakes on vancouver island. Journal of Geophysical Research: Solid Earth, 114(B2), 2009.

[61] Nikolai M Shapiro, Michel Campillo, Edouard Kaminski, Jean-Pierre Vilotte, and Claude Jaupart. Low-frequency earthquakes and pore pressure transients in subduction zones. Geophysical Research Letters, 45(20):11-083, 2018.

[62] D. R. Shelly and J. L. Hardebeck. Precise tremor source locations and amplitude variations along the lower-crustal central san andreas fault. Geophysical Research Letters, 37(14), 2010.

[63] D. R. Shelly, G. C. Beroza, S. Ide, and S. Nakamula. Low-frequency earthquakes in shikoku, japan, and their relationship to episodic tremor and slip. Nature, 442(7099):188-191, 2006.

[64] D. R. Shelly, G. C. Beroza, and S. Ide. Complex evolution of transient slip derived from precise tremor locations in western shikoku, japan. Geochemistry, Geophysics, Geosystems, 8(10), 2007.

[65] D. R. Shelly, G. C. Beroza, and S. Ide. Non-volcanic tremor and low-frequency earthquake swarms. Nature, 446(7133):305-307, 2007.

[66] M Supino, N Poiata, G Festa, JP Vilotte, C Satriano, and K Obara. Self-similarity of low-frequency earthquakes. Scientific Reports, 10(1):1-9, 2020.

[67] Amanda M Thomas, Gregory C Beroza, and David R Shelly. Constraints on the source parameters of low-frequency earthquakes on the san andreas fault. Geophysical Research Letters, 43(4):1464-1471, 2016.

[68] D. J. Thomson. Spectrum estimation and harmonic analysis. Proceedings of the IEEE, 70(9): 1055-1096, 1982.

[69] MJ Tompkins and NI Christensen. Ultrasonic p-and s-wave attenuation in oceanic basalt. Geophysical Journal International, 145(1):172-186, 2001.

[70] Peter E. van Keken, Bradley R. Hacker, Ellen M. Syracuse, and Geoff A. Abers. Subduction factory: 4. depth-dependent flux of h2o from subducting slabs worldwide. J. Geophys. Res., 116 (B1):B01401, 2011. ISSN 2156-2202. doi:10.1029/2010JB007922. URL http://dx.doi.org/10. 1029/2010JB007922 
[71] Emily Warren-Smith, Calum J. Chamberlain, Simon Lamb, and John Townend. High-Precision Analysis of an Aftershock Sequence Using Matched-Filter Detection: The 4 May $2015 M_{1} 6$ Wanaka Earthquake, Southern Alps, New Zealand. Seismological Research Letters, 88(4):10651077, July 2017. ISSN 0895-0695, 1938-2057. doi:10.1785/0220170016. URL https://pubs. geoscienceworld.org/srl/article/88/4/1065-1077/354127.

[72] P. Wessel, W. H. F. Smith, R. Scharroo, J. Luis, and F. Wobbe. Generic mapping tools: Improved version released. Eos, Transactions American Geophysical Union, 94(45):409-410, 2013. ISSN 2324-9250. doi:10.1002/2013E0450001. URL http://dx.doi.org/10.1002/2013E0450001. 